\title{
THE S-BAND LINEAR COLLIDER TEST FACILITY AT DESY
}

\author{
M. Schmitz for the SBLC study group \\ Deutsches Elektronen Synchrotron (DESY), Notkestraße 85, D-22603 Hamburg, GERMANY
}

\section{Abstract}

The S-Band Linear Collider Test Facility under construction at DESY serves as a test bed for the essentiell hardware to build a large scale $\mathrm{e}^{+} \mathrm{e}^{-}$linear collider based on a $3 \mathrm{GHz}$ accelerating $\mathrm{rf}$ and having a center of mass energy of at least $500 \mathrm{GeV}$. Besides the injection system at the beginning and a beam analysis area at the downstream end, the central part of the test facility consists of two modular units similar to those to be installed in a S-Band Linear Collider (SBLC) tunnel. The key-questions of Higher Order Mode (HOM) excitation, measurement, damping and feedback on the alignment of the accelerating structure as well as the quadrupole position have to be answered in order to manage the most crucial aspect of a $15 \mathrm{~km}$ linear accelerator, namely the beam stability. The final layout of this test facility and the performance of the present setup including the first accelerating section fed by a $150 \mathrm{MW}$ klystron will be presented.

\section{INTRODUCTION}

Since 1991 a study group investigates the feasibility of a large S-Band Linear Collider (SBLC). Although S-Band accelerators are used in a lot of laboratories, the demands for a SBLC are not simple extrapolation from any existing accelerator. Therefore a R\&D program started in 1992 to build up a S-Band Test Facility at DESY, in which the crucial aspects for a SBLC design can be investigated experimentally:

- Design, construction and operation of an injection system that is capable to produce bunchtrains that fulfill the SBLC demands (see section II).

- Development, operation and improvement of a high peak power rf-source, i.e. klystron and modulator as well as other high power components, like rf-windows and loads (see section III).

- Elaboration of a reliable concept to keep the HOM-effects below a tolerable limit determined by emittance growth. In this context measurement and damping of HOM's as well as straightness and alignment of the accelerating structure has to be mentioned (see section IV).

- Development of suppression techniques fighting ground motion and vibration (see section V).

The general layout of this test facility is shown in figure 1 and its main parameters are listed in table 1 . Delivered by an injector the beam will be accelerated by 2 modular units similar to those as being proposed for the SBLC.
One module consists of 2 travelling wave sections $(\beta=1$, $2 \pi / 3$-mode, $17 \mathrm{MV} / \mathrm{m}$ ) of $6 \mathrm{~m}$ length each, that are driven by one $150 \mathrm{MW} / 3 \mu$ s klystron connected to a $375 \mathrm{MW}$ peak power modulator. In addition the downstream end of each accelerating section is completed with correctors, one screen and a focussing triplet accomodating a stripline type beam position monitor. Before being dumped at the end of the $40 \mathrm{~m}$ long test facility the quality of the $400 \mathrm{MeV}$ beam will be analyzed using a spectrometer beamline including an OTR screen.

\begin{tabular}{|l|c|l|}
\hline energy at full current & 400 & $\mathrm{MeV}$ \\
\hline injector energy & $\approx 4$ & $\mathrm{MeV}$ \\
\hline length of bunchtrain & $\geq 2$ & $\mu \mathrm{s}$ \\
\hline number of bunches & $1-250$ & \\
\hline bunch to bunch separation & 8,16 or 24 & $\mathrm{~ns}$ \\
\hline particles per bunch & $1.5,3.0$ or 4.5 & $10^{10} \mathrm{e}^{-}$ \\
\hline current in bunchtrain & $\geq 300$ & $\mathrm{~mA}$ \\
\hline normalized rms-emittance & $\approx 10010^{-6}$ & $\pi \mathrm{m} \mathrm{rad}$ \\
\hline
\end{tabular}

Table 1: Main parameters of the S-Band test linac

In the present status the beamline ends downstream of the 1. acc. section behind the focussing triplett. Except for a 4-cell travelling wave structure (TWB1), which is quite important for the process of bunching the injector is completely built up and delivers beam to the first acc. section. Since the first half of this section is surrounded by solenoids, it can not be mounted on the regular girder containing the micro-movers (see section IV). Therefore the concept of beam-based-alignment is not applicable for this section anyhow and thus a $5.2 \mathrm{~m}$ long section similar to the regular $6 \mathrm{~m}$ section (see table 3 ) has been placed there. The additional space will accomodate a kicker by which means the excitation of HOM's can be provoked in the following 3 regular $6 \mathrm{~m}$ sections. From this point of view the 1.acc. section can also be regarded as belonging to the injection system. Results of the beam operation with this present setup are described in the next section.

\section{INJECTOR AND BEAM OPERATION}

The injector, combining high bunchcharge with short interbunch spacing, was designed by means of EGUN and PARMELA calculations [1]. The pulses of desired charge in a train of adequate timestructure are generated at a $90 \mathrm{kV}$ thermionic gun and compressed by means of two standing wave subharmonic bunchers SHB1

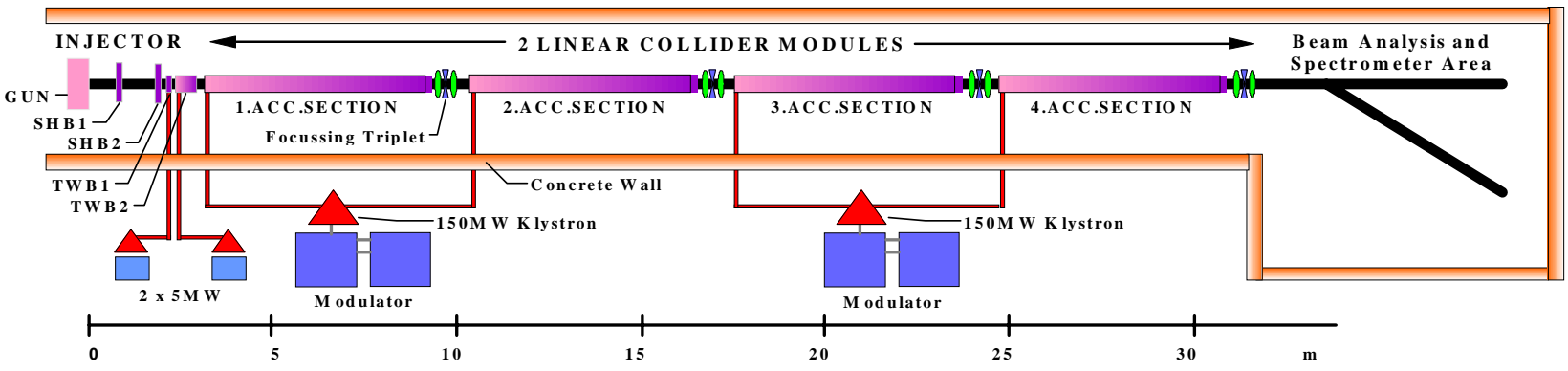

Figure 1: General Layout of the S-Band Test Facility 
$(125 \mathrm{MHz} / 34 \mathrm{kV})$ and SHB2 $(500 \mathrm{MHz} / 36 \mathrm{kV})$ as well as two S-Band travelling wave structures TWB1 $(\beta=0.6$, 4cells, $2 \pi / 3,7 \mathrm{MV} / \mathrm{m})$ and TWB2 $(\beta=0.95,16$ cells, $8 \pi / 9$, $12 \mathrm{MV} / \mathrm{m})$. Two $5 \mathrm{MW}$ klystrons will be used to supply TWB1 and TWB2 individually. In order to achieve a gradient of $12 \mathrm{MV} / \mathrm{m}$ at the available power of $5 \mathrm{MW}$ while maintaining a large iris aperture, the group velocity of TWB2 is only $0.4 \% \cdot \mathrm{c}$. Therefore the $8 \pi / 9$-mode was chosen. Starting with a $90 \mathrm{kV}$ gunpulse carrying $12 \mathrm{nC}$ $\left(7.5 \cdot 10^{10} \mathrm{e}^{-}\right)$in a FWHM-bunchlength of $2.5 \mathrm{~ns}$, PARMELA simulation predicts an overall compression to a pulselength shorter than 10ps with more than $95 \%$ transmission. Since both TWB's also serve for acceleration the kinetic energy at the output of the injector is expected to be about $4 \mathrm{MeV}$.

Starting in april 1995 with a short beamline dedicated for commissioning of the bunchtrain production at the gun, a longer beamline including both SHB's investigated their bunching performance from autumn 1995 to end of june 1996 [2]. The present setup started its operation in autumn 1996. It includes the first accelerating section and the whole injector complex except for TWB1.

The gun produces $2 \mu \mathrm{s}$ long bunchtrains with $8 \mathrm{~ns}$, $16 \mathrm{~ns}$ or $24 \mathrm{~ns}$ interbunch spacing. The gunpulses carry at least $7 \mathrm{nC}\left(4.4 \cdot 10^{10} \mathrm{e}\right)$ in a FWHM length of typically $2.2 \mathrm{~ns}$. Their normalized rms-emittance measured $60 \mathrm{~cm}$ downstream of the gun was $3 \pi \cdot \mathrm{mm} \cdot \mathrm{mrad}$ compared to the PARMELA prediction of $6.8 \pi \cdot \mathrm{mm} \cdot \mathrm{mrad}$. From the same simulations 200ps long pulses are expected after compression with both SHB's. Bandwidth limited by the wallcurrent monitor 300ps long signals and a transmission better than $85 \%$ were observed. This is valid for every bunch of the train, since a feedforward-system compensates beamloading effects at both SHB's. In the final stage TWB1 sitting at the longitudinal focal point determined by the operation of both SHB's and having a phase velocity of $0.6 \cdot \mathrm{c}$ will guarantee a clean capture of these pulses.

However TWB1 is still missing while TWB2 is already at its final position. In order to be captured at TWB2 having a phase velocity of $0.95 \cdot \mathrm{c}$, the $90 \mathrm{keV}$ pulses have to be accelerated at SHB2 while SHB1 still operates around zero crossing. From a $6.8 \mathrm{nC}\left(4.25 \cdot 10^{10} \mathrm{e}\right)$ gunpulse about $5 \mathrm{nC}\left(3.12 \cdot 10^{10} \mathrm{e}^{-}\right)$can be found downstream of TWB2. In that optimized case SHB2 raises the average beamenergy by $36 \mathrm{keV}$ while its amplitude was $52 \mathrm{kV}$ which gives a phase of about $50^{\circ}$ wrt. the beam. Finally $4.8 \mathrm{nC}\left(3 \cdot 10^{10} \mathrm{e}^{-}\right)$have been accelerated through the 1. acc. section of $5.2 \mathrm{~m}$ length. From a $8 \mu \mathrm{m}-\mathrm{Al}$ foil mounted $\approx 1.5 \mathrm{~m}$ behind this section Optical Transmission Radiation (OTR) has been detected and a profile of the beam can be seen. Measuring the displacement of the beam at the OTR-screen introduced by a correction coil results in a beam momentum of $100 \mathrm{MeV} / \mathrm{c}( \pm 15 \%)$, corresponding to an accelerating gradient of $19 \mathrm{MV} / \mathrm{m}$. Taking the $5.2 \mathrm{~m}$ section parameters from table 3 this gradient compares fairly well with the measured input power of 58MW.

Besides the information on the beam profile, the OTR will be used for energy and bunchlength measurement. In the first case its angular distribution has to be analyzed, while in the second case the frequency spec- trum of the infra-red part of the coherent OTR needs to be measured. Both experiments just started commissioning their setup.

\section{MODULATOR AND KLYSTRON}

Since modulators and klystrons contribute significantly to the total cost of a linear collider, as much peak power as possible has to be produced with a single device. In 1993 a R\&D program together with SLAC, the Technical University of Darmstadt, PHILIPS (Hamburg) and DESY started to construct and operate two $150 \mathrm{MW} / 3 \mu \mathrm{s} / 50 \mathrm{~Hz}$ klystrons. Accompanied by 2D and 3D simulations [3] to optimize the overall layout, in 1994 the first and in 1995 the second klystron having a slightly modified output circuit geometry and improved HOM damping in the drift tube was built and tested at SLAC meeting the requirements as can be seen in table 2 .

\begin{tabular}{|l|c|c|c|l|}
\hline & Design & $\# 1$ & $\# 2$ & \\
\hline beam voltage & 535 & 527 & 508 & $\mathrm{kV}$ \\
\hline$\mu$-perveance & 1.79 & 1.78 & 1.80 & $\mathrm{~A} / \mathrm{V}^{1.5}$ \\
\hline output power & 150 & 153 & 150 & $\mathrm{MW}$ \\
\hline pulse length & 3.0 & $\geq 3.0$ & $\geq 3.0$ & $\mu \mathrm{s}$ \\
\hline efficiency & 40 & 43 & 45 & $\%$ \\
\hline gain & $\geq 50$ & 56 & 57 & $\mathrm{~dB}$ \\
\hline
\end{tabular}

Table 2: Parameters of both 150MW klystrons

Similar to the one at SLAC to test the $150 \mathrm{MW}$ klystron at full power and $60 \mathrm{~Hz}$, two PFN-type modulators for the test facility are built at DESY. They consist of 4 lines in parallel resonantly charged up to $50 \mathrm{kV}$ within $17 \mathrm{~ms}$ via a charging choke. A current of $16 \mathrm{kA}$ is supplied into the primary of a 1:23 pulse transformer when discharging the PFN by switching two thyratrons. Maximum ratings of the modulator are $535 \mathrm{kV}$ and $700 \mathrm{~A}$ [4].

The first unit of modulator and klystron has been installed in the test facility and was successfully commissioned at $150 \mathrm{MW} / 3 \mu \mathrm{s} / 50 \mathrm{~Hz}$ with the same performance as during the SLAC tests (see table 2). The second unit will be commissioned soon.

Since November 1996 one output arm of the $150 \mathrm{MW}$ klystron\#1 has been connected to the 1 . acc. section while the other still works on a load. Presently, after about 400h of beam operation and conditioning, the section can be operated with an $1.5 \mu \mathrm{s}$ long rf-pulse of $60 \mathrm{MW}$ (i.e. $120 \mathrm{MW}$ of klystron power) at $25 \mathrm{~Hz}$ repetition.

\section{ACC. SECTION AND HOM-HANDLING}

The accelerating section is of constant gradient type operating in $2 \pi / 3$-mode, with a continuous taper of the group velocity along its whole length of $6 \mathrm{~m}$. HOM handling to avoid emittance dilution due to single- and multibunch instabilities together with simple construction techniques to reduce the costs for mass production are the main topics to be investigated. The HOM handling concept requires HOM-damping, adequate section detuning as well as a certain straightness and alignment of the section. Assuming an average Q-value of the HOM's of 4000 (natural $\approx 13000$ ) and the SBLC bunchcharge of $1.8 \mathrm{nC}$ (6ns interbunch spacing) the total accuracy of alignment and straightness of the $6 \mathrm{~m}$ section has to be $\leq 50 \mu \mathrm{m} \mathrm{rms}$. In that case beam dynamics calculations predict an emittance growth of about $20 \%$ for a $15 \mathrm{~km}$ long linac. 
Since there are a lot of trapped modes in long travelling wave structures, external dampers would have to be coupled to the section at many positions, which is neither reasonable nor cost saving. Instead of that internal damping by sputtering a thin $(20 \mu \mathrm{m})$ steel layer onto the iris showed a Q-reduction of the HOM by a factor of 5, while the fundamental Q changes only by $5 \%$. This layer withstood an iris tip field of $28 \mathrm{MV} / \mathrm{m}$ in a high power standing wave test resonator without any degradation.

Nevertheless one or two cells will be equipped with HOM couplers to measure the beam induced power which is correlated to the average excentricity of the beam axis wrt. the section. This signal has to be minimized (beam based alignment) when driving the micromovers that are mounted below the section girder. The girder as shown in fig. 2 must not deteriorate the straightness of the section, mounted on top of it, even with temperature transient around it. Therefore it is constructed from a tube ( $448 \mathrm{~mm}$ dia.) that is totally thermal insulated. The movers, one on each end of the girder can operate between $\pm 1.5 \mathrm{~mm}$ with a step size of $150 \mathrm{~nm}$ (see figure 2).

\begin{tabular}{|l|c|c|l|}
\hline & Linac II & SBLC & \\
\hline length of section & 5.2 & 6 & $\mathrm{~m}$ \\
\hline attenuation & $0.5-0.6$ & 0.55 & neper \\
\hline group velocity & $3.3-1.2$ & $4.1-1.3$ & $\% \cdot \mathrm{c}$ \\
\hline filling time & 750 & 790 & $\mathrm{nsec}$ \\
\hline
\end{tabular}

Table 3: Parameters of the accelerating sections

for LINAC II and SBLC

In the process of replacing old sections of the LINAC II (the $\mathrm{e}^{+} \mathrm{e}^{-}$injector linac at DESY) $5.2 \mathrm{~m}$ long sections having comparable parameters (see table 3) were built at DESY. While the straightness tolerance is easily met after the vertical inductive brazing of typically $1 \mathrm{~m}$ long pieces, which have a measured rms deviation of typically $20 \mu \mathrm{m}$, the horizontal braze of the complete $5.2 \mathrm{~m}$ section has shown deviations of up to $1 \mathrm{~mm}$. They have been decreased down to $100 \mu \mathrm{m}$ rms by a recently developed straightening procedure, and will achieve the target value of $30 \mu \mathrm{m}$ after commissioning of an improved straightening device constructed for this reason.

Up to now five $5.2 \mathrm{~m}$ sections have been assembled, tuned and high power tested at DESY. One of them is installed in the test facility as 1 . acc. section and 3 are operating in the LINAC II continuously without difficulties.

Other major technical developments being made so far are a very compact symmetric high power input cou-

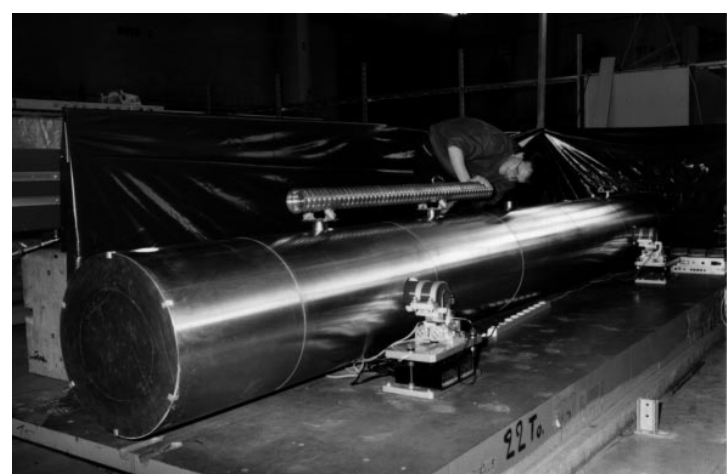

Figure 2: Regular girder for the $6 \mathrm{~m}$ section including micro movers (short piece of structure on top to demonstrate the full assembly) pler [5] and the collinear load [6]. The collinear load absorbs the remaining rf-power over the last eight cells of the section while still accelerating the beam. Such a load avoids a second high power coupler (costs), represents no obstacle for the inductive brazing coil, is perfectly symmetric (no transverse kicks due to field asymmetries) and absorbs any higher order mode touching the end of the section.

The first $6 \mathrm{~m}$ section equipped with symetric high power couplers, iris coating, internal load and $2 \mathrm{HOM}$ couplers (one at the front end and one almost at $2 / 3$ of the section length) will be installed on the regular girder in the test linac end of May this year.

\section{GROUND MOTION AND VIBRATION}

Any kind of quadrupole motion within the frequency range of $2-30 \mathrm{~Hz}$ can hardly be damped either in a passive manner or with beam based feedback techniques. Therefore ground motion detectors (geophones and accelerometers) have been tested and further developed [7]. Each quadrupole in the test facility will be equipped with such a detector to feed back on the vertical quadrupole position via piezo movers. Attenuation of amplitudes up to $14 \mathrm{~dB}$ within this frequency range has been achieved and corrects the vertical rms quadrupole motion down to the $20 \mathrm{~nm}$ range.

In addition a simple and stiff concrete support with mechanical resonances well beyond $100 \mathrm{~Hz}$ has been built for the quadrupole to avoid any externally driven excitation. To decouple the vibration introduced by water flow within the coil windings of the quadrupole, the coils are mounted on a separate aluminium support within the quadrupole yoke. This support can be mounted separately to the floor.

\section{ACKNOWLEDGEMENT}

I would like to thank all the members of the collaboration from the different institutes and countries for their contributions. The significant progress which has been made during the last 5 years is based on the enthusiasm of the people involved and the willingness of the management to encourage this work.

\section{REFERENCES}

[1] M. Schmitz, A.D. Yeremian, "The Injector for the S-Band Test Linac at DESY", Proc. of the 1994 International Linac Conference, Tsukuba, Japan, 21-26 August 1994

[2] M. Schmitz, "Performance of the first Part of the Injector for the SBand Test Facility at DESY", Proc. of the 1996 International Linac Conference, Geneva, Switzerland, 26-30 August 1996

[3] M. Dohlus et al, "Comparison of CONDOR, FCI and MAFIA Calculations for a 150 MW S-Band Klystron with Measurements", Proc. of the 1995 P.A.C., Dallas, Texas, 1-5 May 1995

[4] S. Choroba et al., "A 375MW Modulator for a 150MW Klystron at the S-Band Linar Collider Test Facility at DESY", Proc. of the 1996 International Linac Conference, Geneva, Switzerland, 26-30 August 1996

[5] V.E. Kaljuzhny et al, "Design and Performance of a Symmetric High Power Coupler..", DESY-M-94-11, 1994, Internal Report

[6] J. Haimson, "Collinear Termination for High-Energy Particle Linear Accelerators", PATENT-US-3264515, Aug 1966. 7pp.

[7] C. Montag, "An active mechanical Stabilization System for Linear Collider Quadrupoles to compensate fast Ground Motion", Proc. of the 1996 International Linac Conference, Geneva, Switzerland, 2630 August 1996 